
allemande

\title{
Un médecin roumain de Transylvanie face à l'occupation allemande de 1917-1918: les Notes du Dr Vasile Bianu (1858-1927)
}

Ligia Livadă-Cadeschi

\section{(2) OpenEdition \\ Journals}

Édition électronique

URL : https://journals.openedition.org/allemagne/582

DOI : 10.4000/allemagne.582

ISSN : 2605-7913

Éditeur

Société d'études allemandes

Édition imprimée

Date de publication : 29 décembre 2017

Pagination : 393-406

ISSN : 0035-0974

\section{Référence électronique}

Ligia Livadă-Cadeschi, « Un médecin roumain de Transylvanie face à l'occupation allemande de 1917-1918 : les Notes du Dr Vasile Bianu (1858-1927) », Revue d'Allemagne et des pays de langue allemande [En ligne], 49-2 | 2017, mis en ligne le 29 décembre 2018, consulté le 18 mai 2021. URL http://journals.openedition.org/allemagne/582 ; DOI : https://doi.org/10.4000/allemagne.582 


\section{Un médecin roumain de Transylvanie face à l'occupation allemande de 1917-1918: les Notes du Dr Vasile Bianu (1858-1927)}

\section{- Ligia Livadă-Cadeschi *}

Les Notes de la guerre de la Grande Roumanie (Însemnările din Războiul României Mari) publiées en 1926 par le Dr Vasile Bianu sont sans doute l'un des exemples les plus représentatifs de mémoires historiques jamais écrits par un membre du corps médical roumain. Si dans les mémoires autobiographiques l'accent est mis sur l'histoire de la vie de l'auteur, le principal ressort des mémoires historiques est l'histoire ${ }^{(1)}$. Les Notes répondent à la fois à la logique individuelle, personnelle de celui qui les met sur le papier, et à une logique politique. D’ailleurs, presque tous les médecins qui écrivent leurs mémoires historiques sont aussi, au moment de la publication de leurs textes, des hommes politiques ${ }^{(2)}$. Le Dr Vasile Bianu ne fait pas exception à la règle; il signe ses mémoires en qualité tant de médecin que de sénateur de Huedin. Les mémoires historiques sont un instrument de légitimation de la position sociale de leurs auteurs, mais l'État renforce son idéologie historique nationale en publiant, à l'occasion de commémorations, de pareils témoignages ${ }^{(3)}$. Là encore, le Dr Vasile Bianu ne fait pas exception : son ouvrage sera publié avec l'appui du ministre de l'Instruction publique, un autre méde$\operatorname{cin}^{(4)}$, le Dr Constantin Angelescu, à qui l'auteur exprime sa profonde reconnaissance ${ }^{(5)}$.

* Professeur à la Faculté de Sciences Politiques, Université de Bucarest.

1 Constantin BăRbulescu, România medicilor. Medici, ţărani şi igienă rurală în România de la 1860 la 1910, Bucarest, Humanitas, 2015, p. 56.

2 Ibid., p. 59.

3 Ibid., p. 60 .

4 Pour la relation entre médecine et politique dans l'espace roumain, voir Ligia LIVADĂ-CADESCHI, «Medicină și politică în periodicul Călăuza Sanitară și Igienică», București, 1899-1907, Studia Politica. Revista Română de Ştiinţă Politică, XIV/3 (2014), p. 539-557.

5 Dr Vasile Bianu, Însemnări din Războiul României Mari (Notes de la guerre de la Grande Roumanie), Cluj, Institutul de Arte Grafice Ardealul, t. I, 1926, p. 10. 
La Première Guerre mondiale offrait aux mémorialistes roumains un potentiel inépuisable. Son interprétation s'inscrit dans une vraie mythologie, puisqu'elle constitue le cadre de l'acte fondateur de la Roumanie moderne ${ }^{(6)}$. D'autre part, l'éclatement de la Première Guerre mondiale exacerba le patriotisme des peuples européens et des individus. Moins réfléchis, plus extrémistes qu'à l'ordinaire, ils se mirent à vénérer leurs alliés et à détester leurs ennemis. Ces sentiments étaient alimentés par des gouvernements qui n'avaient de cesse de parer l'allié de toutes les vertus et d'accabler l'adversaire des pires défauts ${ }^{(7)}$.

Les Notes du Dr Bianu sont une monumentale construction idéologique qui trouve son prétexte dans le journal tenu par leur auteur au fil des événements. Mais dans l'économie de l'ouvrage les notes sont largement dépassées par la profusion de fragments d'articles de presse, de discours, de journaux de front, voire d'amples passages d'œuvres historiques et/ou littéraires. Les traits subjectifs, individuels, tiennent soit à la profession de médecin que l'auteur continua d'exercer au sein de l'armée roumaine, soit à son fort sentiment patriotique. Les Notes forment un ouvrage de propagande, où l'épopée nationale l'emporte sur toutes les autres lectures possibles, acquérant souvent des accents mystiques et messianiques. L'axe fondamental selon lequel les événements s'enchaînent n'est pas la guerre en soi, mais l'accomplissement de l'idéal national de la Grande Roumanie. Les Notes ne prennent pas fin avec la guerre ou avec la participation de leur auteur à la guerre, mais avec ce «miracle des miracles » ${ }^{(8)}$ qu'est le couronnement du roi Ferdinand à Alba-Iulia en octobre 1922 - consécration symbolique, subséquente à l'entérinement juridique des traités de paix, de la réalisation de l'unité nationale des Roumains. Sous la plume du Dr Bianu, l'idéal national devient une véritable religion laïque. Avec la «sainte guerre d'unification nationale» ${ }^{(9)}$, «la Roumanie aura vécu l'heure de son salut" ${ }^{(10)}$. Les Notes sont divisées en deux tomes: le Calvaire et l'Apothéose de la nation roumaine ${ }^{(11)}$. L'auteur avoue que, au moment où la mobilisation fut décrétée, «je ressentis le frisson d'une émotion forte et sacrée » ${ }^{(12)}$. Le sacrifice national revêt un caractère profondément festif, unanimement assumé:

«J'eus le plaisir de voir avec quel enthousiasme nos soldats accouraient de partout vers les points de concentration [...]. On eût dit que tous ces héros de la nation allaient à des noces» ${ }^{(13)}$.

Les accents messianiques accompagnent aussi l'évocation des moments dramatiques que connut la Roumanie pendant la guerre. La paix brutalement conclue à Bucarest en mai 1918 fait l'auteur s'exclamer: «Nous attendons la résurrection de notre paix,

6 Lucian Boia, Primul Război Mondial. Controverse, paradoxuri, reinterpretări, Bucarest, Humanitas, 2014, p. 66.

7 Stanislav Sretenovic, «Expressions et usages de la francophilie serbe pendant la Première Guerre mondiale», in: Florin ŢuRCANu (dir.), Francophilie et germanophilie en Europe sud-orientale à la veille et pendant la Première Guerre mondiale, Bucarest, Ed. Universităţii din Bucureşti, 2015, p. 88.

8 Dr V. Bianu, Însemnări (note 5), t. II, 1926, p. 463.

9 Ibid., t. I, p. 10.

10 Ibid.

11 Ibid., p. 8.

12 Ibid., p. 11.

13 Ibid., p. 19-20. 
crucifiée ces jours-ci!» ${ }^{(14)}$. Et, en achevant sa démarche dans la lettre et l'esprit des textes religieux, le Dr Bianu l'intègre à une liturgie politique:

«Quant à moi, humble écrivain, [...] je clorai [...] par les paroles du vieux Siméon: Maintenant, ô Maître, tu laisses ton serviteur s'en aller dans la paix, selon ta parole. Car mes yeux ont vu le salut du peuple roumain! Gloire et louange à Dieu pour les siècles des siècles, amen!» ${ }^{(15)}$.

Mais pour comprendre la démarche fortement légitimante du point de vue national assumée par le Dr Vasile Bianu, il convient de donner quelques précisions sur ses origines transylvaines à une époque où la Transylvanie faisait partie de l'Autriche-Hongrie, ainsi que sur la place privilégiée que la Transylvanie occupe dans l'imaginaire historique roumain. Vasile Bianu naquit en 1858 à Aiud, dans le département de Târnava-Mică. Après avoir terminé son lycée à Blaj, il passa en Roumanie et obtint son doctorat en médecine à Bucarest en 1881. Après des stages de chirurgie à Paris (1885) et à Londres (1886), il s'établit comme médecin à Horezu-Vâlcea (1888-1891), puis à Buzău (1892-1919) ${ }^{(16)}$. Mobilisé en août 1916, il servit dans plusieurs hôpitaux de Galaţi, Buzău, Fălticeni et Iaşi, où il arriva à la suite de l'ordre d'évacuation d'août 1917. Démobilisé en septembre 1918, il revint à Buzău. Il mourut en 1927 à Cluj, où il s'était fixé après la guerre.

Pour le Dr Bianu, le choix d'être citoyen roumain, dans un État roumain, est catégorique, définitif et bien antérieur à l'éclatement de la guerre; il se dit prêt à ignorer les ordres militaires au cas où on lui ordonnerait de rester, en sa qualité de médecin militaire, sur le territoire occupé:

«L'idée me tourmentait de rester sur place, à Fălticeni, en cas d'évacuation, de rester sous la domination des Allemands et des Hongrois, moi, Transylvain qui, dès l'école primaire, à Blaj, [...] je terminais chaque soir ma prière en demandant au bon Dieu de m'aider à terminer mon lycée et à passer en Roumanie. [...] Si on ne me trouve pas une nouvelle affectation sur le territoire libre de mon pays, je partirai de mon propre chef, au dernier moment, coûte que coûte! ${ }^{(17)}$.

Heureusement, cette éventualité lui fut épargnée.

D’autre part, dans la hiérarchie symbolique des territoires roumains, la Transylvanie brillait plus que tous les autres ${ }^{(18)}$. La culture roumaine moderne et la conscience de l'origine historique et linguistique latine avaient été façonnées dans la plus grande mesure par les érudits transylvains. Dans les représentations historiques roumaines, la Transylvanie était le cœur même de l'organisme national, le centre de l'antique Dacie et l'aire principale de formation du peuple roumain ${ }^{(19)}$. Pour le Dr Bianu, le soldat roumain auquel il dédiait son ouvrage monumental n'était ni plus ni moins que «le représentant de la gloire romaine» ${ }^{(20)}$, «descendant de Rome l'éternelle transplanté dans l'est par le divin Trajan, sentinelle latine aux bouches du

14 Ibid., p. 389-390.

15 Ibid., t. II, p. 495.

16 Ion Negru, «La aniversarea a 60 de ani de la Marea Unire. Însemnările chirurgului Vasile Bianu din războiul pentru întregirea patriei», Viaţa Medicală, vol. XXVI/2, p. 93.

17 Dr V. Bianu, Însemnări (note 5), t. I, p. 160.

18 Lucian BoiA, "Germanofilii». Elita intelectuală românească în anii Primului Război Mondial, Bucarest, Humanitas, 2009, p. 11.

19 Ibid., p. 57.

20 Dr V. Bianu, Însemnări (note 5), t. I, Dedicaţie (Dédicace). 
Danube! » ${ }^{(21)}$. Et l'État roumain lui-même tire sa sève de la terre transylvaine, «foyer et point de départ de Rodolphe le Noir, fondateur de la Valachie ${ }^{(22)}$.

Au niveau de la construction de l'idéologie nationale roumaine, les nuances et les dilemmes individuels se perdent dans le discours majoritaire, devenu officiel avec l'entrée en guerre de la Roumanie et notamment après la constitution de la Grande Roumanie. Ce qui ne se passe cependant pas dans la même mesure et avec la même acuité au niveau de la politique et de la diplomatie concrète de l'époque. Dans les décennies précédant la Première Guerre mondiale, la Roumanie s'était intégrée dans un système complexe de rapports avec les grandes puissances centre-européennes, au sein duquel la problématique des Roumains de Transylvanie (et non de la Transylvanie en tant que province) était bien plus nuancée et moins clamée, en tout cas, de façon moins énergique. Les relations du Royaume de Roumanie avec l'espace allemand (y compris autrichien) incorporaient trois dimensions fondamentales - dynastique, politicodiplomatique, et culturelle -, mais la problématique des Roumains de Transylvanie n'était envisagée que du point de vue de la dernière.

La constitution de l'État roumain moderne est indissolublement liée à la dynastie allemande des Hohenzollern-Sigmaringen. Le roi Carol $\mathrm{I}^{\text {er }}$ (1866-1914), avec un règne de quarante-huit ans, avait été le garant, pour ensuite devenir le symbole, de la modernité et de l'européanité des Roumains. Son choix politique et diplomatique des Puissances centrales (matérialisé par les traités de 1883), assumé par l'élite politique (bien que secret pour l'opinion publique), s'inscrivait dans la logique des garanties de sécurité pour un Royaume de Roumanie indépendant de fraîche date. D'autre part, le prestige culturel de l'espace allemand était important non seulement parmi les Roumains de Transylvanie, mais aussi pour l'élite politique et culturelle de l'ancien Royaume. À ce strict niveau, ceux qui penchent pour la neutralité ou pour une coopération avec les Puissances centrales ne le cèdent en rien à leurs adversaires; ils sont, il est vrai, moins visibles et leur impact est plus limité, mais c'est précisément parce que l'opinion publique est moins réceptive ${ }^{(23)}$. Au niveau de l'opinion publique (il s'agit de personnes possédant une certaine culture et manifestant un intérêt plus ou moins grand pour les affaires publiques), le sentiment dominant était celui de la solidarité avec l'Entente, surtout avec la France, le grand repère culturel de l'élite roumaine ${ }^{(24)}$. Cependant, les germanophiles n'étaient ni rares, ni dépourvus d'influence ${ }^{(25)}$. En plus, si l'ensemble des membres de la classe supérieure a été élevé dans le culte de la latinité, dans l'amour de la culture française, il n'en va pas de même au point de vue politique. La France républicaine et anticléricale n'est pas du goût des conservateurs ni même de la plupart des libéraux. Certes, beaucoup de Roumains respectent sincèrement l'ordre et la force de l'Allemagne, pays qui leur a donné le roi; mais la plupart de ceux qu'on appelle «germanophiles» sont en fait des «russophobes» ${ }^{(26)}$.

21 Ibid., t. II, p. 108.

22 Ibid., t. I, p. 29.

23 L. BoiA, «Germanofilii» (note 18), p. 61-62.

24 L. BoiA, Primul Război Mondial (note 6), p. 69.

25 Ibid., p. 71-72.

26 Jean-Noël Grandhomme, «Les élites roumaines et la France dans la période de satellisation de la Roumanie par les Puissances centrales (décembre 1917-novembre 1918)», in: Fl. ȚURCANU (dir.), Francophilie et germanophilie (note 7), p. 161. 
Le Dr Bianu est un représentant typique de l'opinion publique de son époque, opinion publique dont le diplomate Ioan C. Filitti disait en septembre 1914 qu'elle était dominée par la sympathie pour la France et la haine contre les Hongrois ${ }^{(27)}$. D’autre part, n'oublions pas que les Notes furent publiées bien après la fin de la guerre, alors que l'idéal national avait triomphé et que la Grande Roumanie était devenue une réalité. Bien qu'elles répondent clairement à la logique d'unanimité de l'idéal national officiel, elles laissent percevoir entre les lignes les échos de dilemmes qui peuvent avoir taraudé leurs contemporains. La discussion des rapports avec l'espace allemand n'est pas sans nuances: l'auteur souligne à plusieurs reprises l'antagonisme irréconciliable entre la culture allemande et l'appétit guerrier des États allemand et austro-hongrois. Mais le Dr Bianu envisage les rapports de la Roumanie avec les Puissances centrales sous l'angle de l'idéal national roumain, dissociant l'État allemand constitué par unification sur le principe des nationalités (ce qui pouvait le rapprocher de la situation de l'État roumain) et l'État multinational austro-hongrois, visiblement réticent à reconnaître des droits égaux aux différentes nationalités qui le composaient. Selon cette formule, l'État allemand était pour la Roumanie non seulement un modèle d'État issu de l'unification de territoires habités par des populations parlant la même langue, mais aussi un garant des droits des différentes nationalités composant le troisième partenaire, l'Autriche-Hongrie. C'est pourquoi, en évoquant la déclaration de guerre remise par la Roumanie à l'Autriche-Hongrie en août 1916, le Dr Bianu cite le passage renvoyant à cette position de l'Allemagne, dans laquelle les Roumains avaient vu un possible soutien des droits nationaux des Transylvains:

«En adhérant, en 1883, au groupe des Puissances centrales, la Roumanie [...] avait vu dans les rapports d'amitié et d'alliance établis entre les trois grandes puissances un gage précieux pour sa tranquillité intérieure, aussi bien que pour l'amélioration du sort des Roumains d'Autriche-Hongrie. En effet, l'Allemagne et l'Italie, qui avaient reconstitué leurs États sur la base du principe des nationalités, ne pouvaient pas ne pas reconnaître la légitimité du fondement sur lequel reposait leur propre existence ${ }^{(28)}$.

Des efforts allemands dans le sens désiré par les Roumains avaient cependant été faits. Ils étaient bien connus au plus haut niveau, mais le roi Ferdinand (1914-1927) luimême dénonçait leur incapacité à tempérer l'intransigeance austro-hongroise, « déclarant qu'il était fermement convaincu que ni l'Allemagne ni personne ne pouvait rien faire à ce sujet ${ }^{(29)}$. Si l'Autriche-Hongrie a été perçue comme un réel danger du point de vue national, l'image de l'Allemagne s'est progressivement détériorée, au rythme des événements militaires qui conduiront à l'instauration du régime d'occupation.

Le fait d'avoir opté en faveur de l'accomplissement de l'idéal national roumain valut au roi Ferdinand une énorme popularité parmi ses sujets, et revêtit le sens d'une roumanisation totale de la dynastie d'origine allemande qui avait indissolublement lié son nom à la constitution de l'État roumain moderne. Pour le Dr Bianu, l'allusion publique du roi au fait d'avoir vaincu ses propres origines représentait «le meilleur indice qu’il

27 Daniel CAIn, «Identité culturelle et responsabilités: le corps diplomatique roumain et bulgare pendant la Grande Guerre», in: Fl. ŢURCANU (dir.), Francophilie et germanophilie (note 7), p. 127.

28 Dr V. Bianu, Însemnări (note 5), t. I, p. 14.

29 Ibid., p. 17. 
avait pris la bonne voie, qu'il avait choisi le meilleur chemin pour la Roumanie» ${ }^{(30)}$. Le roi avait lui-même répondu à ceux qui soutenaient qu' «une Russie victorieuse ne tolérerait pas une dynastie allemande sur le trône de la Roumanie, [...] qu'il ne pouvait pas séparer les intérêts de la dynastie d'avec ceux du pays, et que la dynastie n'était pas allemande, mais roumaine ${ }^{(31)}$.

Élaborées sous le signe de l'idéal national, les Notes du Dr Bianu ne mettent jamais en question la légitimité de la participation de la Roumanie à la guerre aux côtés de l'alliance franco-britannique qui s'était engagée à reconnaître les droits de l'État roumain sur la Transylvanie, même dans les moments les plus difficiles du conflit. Bien qu'il enregistre sèchement plusieurs cas d'automutilation parmi les hommes de troupe (dans le but de se soustraire aux obligations militaires), l'auteur met l'accent sur l'adhésion inconditionnelle des soldats à la cause nationale:

«D’habitude, nos blessés supportaient avec beaucoup de résignation et de fermeté les douleurs des opérations et des pansements [...] aucune manifestation de la douleur ne contenait la moindre allusion méchante à notre guerre sacrée ${ }^{(32)}$.

La guerre est légitimée par le droit des peuples à l'autodétermination:

«Toutes les grandes puissances du monde [...] suivies par une multitude de peuples plus petits [...] font et feront la guerre jusqu'au moment où [...] chaque nation aura retrouvé son unité et se gouvernera selon sa propre volonté» ${ }^{(33)}$.

Les évolutions dramatiques sur le front roumain et l'imminence du danger allemand détermineront une radicalisation du discours du Dr Bianu. Alors que, pendant la première phase de la participation de la Roumanie à la guerre, il reprochait à l'Allemagne son incapacité à modérer la position de l'Autriche-Hongrie par rapport aux Roumains de Transylvanie (ce qui, somme toute, permettait de justifier l'abandon des alliés de 1883 et le passage dans le camp adverse), à mesure que s'annonce le désastre militaire roumain, où le rôle de l'armée allemande est déterminant, le Dr Bianu dénonce le militarisme allemand et ses rêves de domination universelle. Au printemps de 1917, dans le contexte de l'occupation allemande d'une grande partie de l'ancien Royaume, ainsi que de la mondialisation du conflit, ses accusations à l'adresse de l'Allemagne sont formulées en des termes très crus comme la criminalité tudesque, l'insolence germanique, le danger teutonique, la barbarie allemande. Au-delà des duretés de langage, pour le Roumain transylvain les différences entre l'Allemagne et l'Autriche-Hongrie du point de vue de l'opposition entre principe national et multinational doivent conditionner le traitement différent de ces deux grandes puissances centre-européennes à la fin de la guerre. C'est sans doute ce qui lui fait insérer dans le texte des Notes un discours prononcé au Parlement en juillet 1917 par le conservateur-démocrate Take Ionescu, discours qu'il considère comme étant d'une rare beauté:

«L'Allemagne a commis le plus grave crime de l'histoire. Mais, en même temps, elle s'est manifestée comme une puissance [...] Il est impossible, il est injuste de refuser à la nation allemande l'unité que nous réclamons pour une autre. Mais ce serait le comble de la folie de

30 Ibid., p. 14.

31 Ibid., p. 16.

32 Ibid., p. 45.

33 Ibid., p. 8. 
laisser à l'Allemagne un État vassal, un État artificiel de cinquante-deux millions d'habitants, qui lui fournisse une réserve humaine pour un nouvel attentat à la liberté des nations. L'État artificiel de l'Autriche doit être aboli » ${ }^{(34)}$.

Les paix séparées signées en Europe de l'Est sont interprétées comme preuves des intentions allemandes de domination politique et économique dans cette partie du monde, sans aucun rapport avec l'unité politique du peuple allemand - la seule légitimation qu'accepte le Dr Bianu:

«Tout le monde voit que la paix imposée à la Russie et la paix qu'on veut nous imposer révèlent brutalement la méthode et les buts de l'Allemagne dans cette guerre. La méthode consiste à user de la force sans aucun scrupule [...]. Les buts sont: les conquêtes territoriales, l'asservissement économique des peuples et la création de pays tributaires [...]. En réalité, il n'a jamais été question de défendre la patrie allemande et l'unité politique du peuple allemand. Ç’a été une guerre d’agression préméditée [...]. La victoire allemande à l'Est a rendu un fier service à l'humanité en lui révélant la menace de l'impérialisme envahissant qui inspire la classe dirigeante de l'Allemagne ${ }^{(35)}$.

Militariste et ambitionnant de dominer le monde, l'Allemagne se rend en plus coupable d'hypocrisie et de duplicité politique. La journée du $1^{\mathrm{er}}$ mai 1917 , prétendue fête de l'internationale, donne au Dr Bianu l'occasion de noter:

«Les Allemands furent les défenseurs les plus acharnés et les plus fervents du socialisme cosmopolite et fraternisateur, eux qui déchaînèrent sur l'humanité cette guerre sans égale dans l'histoire du monde. On sait que leurs socialistes ont voté à l'unanimité au Parlement de l'Empire tous les crédits demandés pour la guerre; il est donc naturel de se demander si les Allemands ont été des socialistes internationaux par conviction ou simplement par calcul, pour tromper leurs voisins naïfs et leurs adeptes de par le monde! ${ }^{(36)}$.

Les ambiguïtés du socialisme allemand et celles des rapports entre la Russie et l'Allemagne se potentialisent réciproquement. En évoquant la situation de la Russie de juillet 1917 et ses répercussions sur le front, le Dr Bianu constate que les propagandes allemande et bolchevique sont deux volets de la même conspiration destinée à assurer la suprématie de l'Allemagne dans l'est de l'Europe:

«Le succès de nos ennemis n'est pas dû à leur supériorité, mais en tout premier lieu à l'armée russe qui s'est laissée influencer négativement par la propagande allemande et par celle des maximalistes [...] qui en russe s'appellent bolchevistes, sous la direction de Lénine. [...] L'arrestation de Lénine et de ses complices, instruments de l'Allemagne, vaut une victoire [...], c'est un coup mortel porté à l'odieuse conspiration ourdie dans le but d'agenouiller la Russie devant le bourreau de l'humanité, Guillaume II » ${ }^{(37)}$.

À partir de l'automne 1917, le Dr Bianu insère dans ses Notes un certain nombre de nouvelles sur les troubles socio-politiques ou militaires du camp adverse qui retiennent son attention parce qu'elles annoncent l'effondrement des ennemis de la Roumanie. Il s'agit de désertions de l'armée austro-hongroise (Serbes, «Yougo-slaves ", Tchèques ${ }^{(38)}$,

\footnotetext{
34 Ibid., p. 142-143.

35 Ibid., p. 346-347.

36 Ibid., p. 116.

37 Ibid., p. 154-156.

38 Ibid., p. 225.
} 
Italiens ${ }^{(39)}$ ) qui laissent voir que «la décomposition de l'Autriche-Hongrie prend des proportions considérables » ${ }^{(40)}$, de mutineries dans les armées et les flottes allemandes et austro-hongroises, qui démontrent que «l'état d'esprit tant de la population que de l'armée devient de plus en plus dangereux » ${ }^{(41)}$, ou des grèves d'Autriche, de Prague et de Budapest de janvier $1918^{(42)}$. La décomposition des deux empires centre-européens à l'automne de 1918 est en tout premier lieu le résultat du non-respect du principe des nationalités: "L'Allemagne en tant qu'empire s'est effondrée à jamais. La guerre de 1870 lui a été fatale [...]. Bismarck n'a compris le droit national que du point de vue des Allemands » ${ }^{(43)}$. La même grille de lecture s'applique à l'Autriche-Hongrie:

«Le voilà effondré à jamais, l'empire d'Autriche et, avec lui, la monarchie austro-hongroise [...] qui a commis les pires crimes contre ses nationalités, au gré des intérêts momentanés et changeants des Habsbourg» ${ }^{(44)}$.

En dehors du discours politique et idéologique sur la guerre, dominant dans l'économie de l'ouvrage, les Notes du Dr Bianu contiennent des mémoires, des portraits individuels, des descriptions de places, des évocations d'événements ou de sentiments personnels. Mais leur constante reste le sentiment patriotique, avec parfois des touches nationalistes, voire xénophobes. Le paradigme regroupant invariablement ceux qui ont essayé d'envahir le territoire des Roumains, non seulement en 1918 mais pendant toute leur histoire, est celui des barbares. Les Bulgares, les Turcs et les Allemands sont, dès leur entrée dans la Dobroudja, traités de barbares ${ }^{(45)}$. La barbarie des derniers est d'autant plus condamnable et difficile à comprendre que l'image de l'Allemand dans l'espace culturel roumain était positive ${ }^{(46)}$, valorisant l'attachement des Allemands à l'ordre et au respect des hiérarchies, ainsi que leur appartenance à une culture réputée supérieure. Le Dr Bianu appartient au grand nombre de Roumains qui, bien avant l'entrée en guerre de la Roumanie, avaient dénoncé sans équivoque l'antagonisme entre la culture et le militarisme allemands. Pour Nicolae Iorga, figure mobilisatrice du nouveau nationalisme roumain qui se cristallise après 1900, investi des dimensions d'une figure quasi mythique à l'époque de la Grande Guerre, lorsqu'il fut l'une des âmes de la résistance militaire roumaine de l'année $1917^{(47)}$, distinguer entre monde intellectuel et élites politiques fait partie de son propre combat en Roumanie:

39 Ibid., p. 334-335.

40 Ibid., p. 225.

41 Ibid., p. 238.

42 Ibid., p. 308-309.

43 Ibid., t. II, p. 110.

44 Ibid., p. 119-120.

45 Ibid., t. I, p. 31.

46 Pour l'image de l'Allemand dans l'espace roumain, voir Hans GeHL, «The Germans' Image as Seen by their Romanian, Hungarian and Serbian Neighbours », Philologica Jassyensia, V/2 (2009), p. 103-130.

47 Fl. Țurcanu, «Pourquoi nous aimons la France? L’historien Nicolae Iorga ou du bon usage de la francophilie à l'époque de la neutralité roumaine (1914-1916) », in: Fl. ȚurCANu (dir.), Francophilie et germanophilie (note 7), p. 72-73. 
«Il faut être muni d'une structure mentale tout à fait particulière pour faire un procès à Goethe, Schiller, Kant ou encore Beethoven à cause des actions du général Bissingen [von Bissing] à Bruxelles» (décembre 1915) ${ }^{(48)}$.

Déjà radicalisé après le désastre militaire de la Roumanie, le Dr Bianu n’hésite pas à souligner dans des notes sarcastiques la contradiction entre la supériorité de la culture allemande et les horreurs de la guerre. L’efficacité, mesurée en morts et blessés, des bombardements et de la technique militaire allemands vient «confirmer une fois de plus que la Kultur allemande est la plus parfaite du monde» ${ }^{(49)}$, alors que l'héroïque Belgique aura connu la première les retombées de la civilisation allemande ${ }^{(50)}$. Il affirme qu'il existe un antagonisme irréductible entre la supériorité culturelle et l'agression militaire, réfutant toute justification de celle-ci par celle-là au nom d'une prétendue mission civilisatrice. En juin 1917, la visite officielle du ministre belge Vandervelde à Iaşi, sur le territoire libre, fournit au Dr Bianu l'occasion de formuler un certain nombre d'opinions sur les similitudes entre les destins politiques de la Belgique et de la Roumanie ${ }^{(51)}$, du point de vue de la cause des petits États qui contribuent à la civilisation générale et aux progrès de l'humanité. Le Dr Bianu reprend au discours du ministre belge un passage sur:

«Les coalitions européennes contre Napoléon I ${ }^{\mathrm{er}}$ au siècle passé et contre Guillaume II aujourd'hui. [...] L’armée de Napoléon avait dans ses bagages le Code civil, celle de Guillaume II a dans les siens le manuel de la guerre prussien, avec toutes les horreurs qu'il prescrit et qui furent pratiquées en Roumanie comme en Belgique. Et pourtant, malgré sa gloire, malgré son génie, malgré le rôle historique qu'il a eu, Napoléon a été vaincu par le monde entier, parce qu'il menaçait la liberté du monde » ${ }^{(52)}$.

Les informations de l'auteur au sujet de l'occupation allemande proviennent plutôt de sources indirectes, des communiqués militaires et des rares lettres qui viennent du territoire occupé. Ce qu'il a pu voir de ses propres yeux, c'est surtout le refuge de la population devant les armées des Puissances centrales, refuge dont les autorités auront essayé en vain de diminuer les proportions ${ }^{(53)}$. Les émotions collectives sont alimentées par l'absence d'informations réelles. Déjà, en évoquant un certain nombre de questions concernant l'évolution du front (en novembre-décembre 1916), l'auteur avoue: "tout cela, je l'ai appris par ouï-dire, parce que ça fait quinze jours que nous n'avons plus reçu de journaux et que nous sommes complètement coupés du monde», tandis que les communiqués officiels «sont toujours si brefs et si confus!» ${ }^{(54)}$. Quelques mois plus tard, en janvier 1917, le Dr Bianu revient sur les conséquences psychologiques de l'absence d'informations:

48 Ibid., p. 77.

49 Dr V. Bianu, Însemnări (note 5), t. I, p. 33.

50 Ibid., p. 124.

51 Pour le cliché «la Roumanie, Belgique de l’Orient», voir Laurenţiu VlaD, Pe urmele «Belgiei Orientului». România la expoziţiile universale sau internaţionale de la Anvers, Bruxelles, Liège şi Gand (18941935), Bucarest, Nemira, 2004. 
«Le plus heureux événement est pour nous de recevoir des journaux, quelque vieux qu'ils soient [...]. Souvent, nous oublions même quel jour de la semaine nous sommes, d'autant qu'on nous défend d'indiquer sur les cartes postales que nous envoyons la date et la localité » ${ }^{(55)}$.

Le régime d'occupation allemand est décrit exclusivement par le prisme des vols faits à la richesse nationale roumaine, alors que l'image des rapports entre Allemands et Roumains se construit autour du thème des humiliations que ceux-là font subir à ceuxci. Au-delà de l'image négative que tout occupé se fait naturellement de l'occupant, le discours du Dr Bianu s'inscrit dans une logique politique et idéologique déterminée par les intérêts de la Roumanie à ce moment-là, mais ultérieurement récupérée et perpétuée par le canon de l'historiographie nationale. Le traité de paix séparé de Bucarest, du 7 mai 1918, «n'était pas un diktat, malgré la réputation que lui firent les Roumains, soucieux de maintenir l'actualité des promesses de l'Entente ${ }^{(56)}$. Cette réputation restera fortement ancrée dans la conscience historique des Roumains et ne laissera que peu de place aux nuances et aux réinterprétations. La plupart des historiens roumains dénoncent avec indignation la dureté de l'occupation allemande et le caractère injuste du traité, oubliant que c'était la Roumanie qui avait attaqué les Puissances centrales ${ }^{(57)}$.

Toute l'attitude du Dr Bianu se concentre dans cette exclamation amère qu'il note déjà dans son journal en novembre/décembre 1916: «Nos alliés restent sur place, sans bouger, sur tous les fronts, tandis que nous, nous voyons les Allemands piller à loisir notre beau et riche pays " ${ }^{(58)}$. En février/mars 1917, il insère dans le texte de ses mémoires un communiqué aux troupes roumaines sur le régime d'occupation, qu'il commente amplement, profitant du propagandisme propre à ce type de document. Dans le cadre du thème général du pillage commis par l'occupant, toutes les différences entre les Allemands et leurs alliés sont annulées, tandis que se développe un discours empreint de xénophobie et d’antisémitisme, légitimé par le danger de la dénationalisation des Roumains et de leur destruction comme nation.

«Ces souffrances ont été communiquées aux troupes afin de leur montrer la mauvaise foi de nos ennemis qui utilisent leurs moyens techniques pour nous détruire physiquement et qui veulent la destruction morale de notre armée et de notre population. [...] Le comportement brutal à l'égard de notre pauvre, impuissante population civile [...] est indescriptible et semble démontrer que ces Allemands civilisés sont bien décidés à exterminer le peuple roumain. [...]. Tout cela suffit pour montrer au monde entier qui sont les Allemands et en quoi ils sont différents des Hongrois, des Bulgares et des Turcs. Ajoutons seulement que de toute notre population on n'a épargné que les juifs et les étrangers » ${ }^{(59)}$.

Mais les notes xénophobes portent aussi sur d'autres minorités habitant le territoire de la Roumanie, par exemple: "Tous les Arméniens de chez nous [...] se comportent comme des étrangers à l'égard des Roumains et sont tout aussi germanophiles que les juifs » ${ }^{(60)}$.

55 Ibid., p. 80.

56 Traian SAndu, Histoire de la Roumanie, Paris, Éditions Perrin, 2008, p. 186.

L. Boia, Primul Război Mondial (note 6), p. 77.

58 Dr V. BiAnu, Insemnări (note 5), t. I, p. 61.

59 Ibid., p. 98-99.

60 Ibid., p. 384-385. 
D'autres informations sur le régime d'occupation proviennent de la correspondance, sporadique, avec ceux qui étaient restés sur le territoire occupé. La première lettre, envoyée par l'administrateur de l'hôpital de Buzău, où le Dr Bianu avait travaillé avant sa mobilisation, est de janvier/février 1918. À son propos, le Dr Bianu note:

«Elle est arrivée dans une enveloppe ouverte, sans timbres et sans tampon de la poste, et l'adresse est écrite en allemand [...] afin que l'on voie de quelle liberté jouissent nos frères sous l'administration allemande ${ }^{(61)}$.

En juin 1918, il reçoit une nouvelle lettre de la part du même correspondant, qui lui apprend que

«Tous ceux restés à Buzău sont méconnaissables, tellement ils sont maigres et décatis. [...] Tous les hommes âgés de seize à cinquante ans [...] ont été assignés à différents travaux [...] sans que l'on tienne compte de leur position sociale [...]. Il est impossible de montrer toutes les barbaries commises par ce peuple réputé cultivé et qui prétend être au-dessus de tous les autres» ${ }^{(62)}$.

Un mois plus tard, nous lisons les témoignages de deux amis de l'auteur, avocats et plus ou moins germanophiles. Le premier était resté quelque temps à Bucarest, parmi nos germanophiles; le Dr Bianu le retrouve plus tard à Iaşi et déduit de ses relations que «les Allemands ont fait tout ce qu'on peut imaginer de pire et se sont comportés à l'égard des Roumains de telle sorte que même les germanophiles ont fini par les détester ${ }^{(63)}$. Le second témoignage est une lettre. En la lisant, le Dr Bianu trouve intéressant d'en citer quelques passages:

«"Pour l’instant, ça n’a pas été si mal, parce que nous avons eu de quoi manger - pas comme autrefois, mais enfin, nous n'avons pas souffert de la faim [...]. Moi, j'ai perdu 26 kilos et j'ai maigri comme après dix ans de cure à Marienbad..." Voilà ce que vivent nos pauvres compatriotes dans un pays aussi riche que le nôtre, sous la domination du peuple le plus cultivé et le plus puissant du monde!» ${ }^{(64)}$.

Pour incriminer de nouveau le régime dur imposé par la paix de Bucarest, mais cette fois-ci du point de vue des germanophiles de la classe politique roumaine, le Dr Bianu cite une interpellation du sénateur Victor Miclescu, lequel, tout en reconnaissant la position de vainqueurs des Allemands, dénonce les abus qu'elle a générés:

«Si nous avons accepté la paix des Allemands, je n'entends pas que nous essuyions des vexations continuelles. [...] Le peuple allemand doit apprendre à priser la justice pour tout le monde. Même la presse allemande dit qu'il est impossible d'établir des rapports normaux avec un pays vexé de cette façon ${ }^{(65)}$.

Nous ne savons pas de quels articles parlait le sénateur roumain, mais le Dr Bianu insère, quant à lui, la traduction publiée en juillet 1918 dans Neamul Românesc d'un article de Maximilian Harden paru dans l'hebdomadaire berlinois Die Zukunft, dont il dit:

61 Ibid., p. 323.

62 Ibid., t. II, p. 14-15.

63 Ibid., p. 41-42.

64 Ibid., p. 42-43.

65 Ibid., p. 57. 
«Je regrette que notre censure ait coupé une partie importante de cet article, sans nul doute la plus importante pour nous [...] L'article de Harden se termine ainsi : Le seul résultat de la paix dure est qu'un peuple qui jusque-là penchait vers l'Empire allemand, par admiration amicale, devient amer jusqu'à la haine » ${ }^{(66)}$.

En Roumanie, les Allemands auraient trouvé une partie de l'élite qu'ils voulaient engager dans le processus d'occupation; mais, en dépit d'une relation assez détendue entre occupant et occupé au cours de l'année 1917, l'occupation a produit un désenchantement profond pour une grande partie de la société roumaine, pour les anciens fonctionnaires, pour ceux qui ont connu la pénurie et qui ont volontairement abaissé leur degré de germanophilie ${ }^{(67)}$.

Fin août - début septembre 1918, le Dr Bianu, démobilisé, revient de Iaşi à Buzău. En combinant les informations directes et celles recueillies auprès de ses concitoyens, il brosse un tableau sombre de la situation, dominé par des accusations explicites ou implicites à l'adresse des troupes d'occupation dont le comportement est invariablement qualifié d'abusif.

«Mais ce qui m’a surtout frappé, ç'a été l'aspect lamentable des citoyens [...] dû à l'alimentation insuffisante et au mauvais état d'âme provoqué par l'occupation allemande [...]. Leur souffrance redoublait lorsqu'ils voyaient les Allemands faire bombance, car les autorités et la population étaient obligées de tout mettre à leur disposition, dans des conditions des plus avantageuses et à des prix dérisoires » ${ }^{(68)}$.

L'une des rares relations directes, et qui ne semble pas contaminée par des informations provenant d'autres sources sur des situations ou des événements antérieurs, porte sur la rentrée en guerre de la Roumanie, sur la capitulation de l'Allemagne et sur le retrait des forces d'occupation. Dans le contexte du manque d'informations officielles, ce long passage mêle les opinions politiques de l'auteur et l'évocation de ses propres expériences et sentiments dans une description qui perd sans doute en cohérence, mais gagne en authenticité. Les citoyens de la ville de Buzău ont été "surpris » d'apprendre, en lisant des affiches dans la rue, que l'état de guerre entre l'Allemagne et la Roumanie a été réinstauré:

«Ces nouvelles ont produit une certaine consternation dans le public, qui pour l'instant ne pouvait en comprendre la cause ni en mesurer la teneur. Hier et aujourd'hui, aucun journal n'est arrivé à Buzău, si bien que ça fait deux jours que nous ne savons rien de ce qui se passe de par le monde» ${ }^{(69)}$.

L'impossibilité de s'informer correctement demeure pour le Dr Bianu l'aspect de la vie quotidienne le plus difficile à supporter, et il y revient presque obstinément: «Voilà cinq jours que nous n’avons reçu aucune gazette [...] À présent, même les bruits ont cessé » ${ }^{(70)}$;

66 Ibid., p. 32-33.

67 Alexandru JipA-Teodoros, «La germanophilie roumaine sous l'occupation. Le cas du journal allemand “Bukarester Tagblatt” (décembre 1916-novembre 1918)», in: Fl. ȚurCANu (dir.), Francophilie et germanophilie (note 7), p. 165.

68 Dr V. BIANU, Însemnări (note 5), t. II, p. 62-67.

69 Ibid., p. 103.

$70 \quad$ Ibid., p. 105. 
"Joie immense. Au bout de douze jours d'isolement, nous avons enfin reçu des journaux que nous avons lus d'un seul trait ${ }^{(71)}$.

Le comportement des troupes allemandes en retrait est décrit comme non moins abusif que celui pendant l'occupation, bien que la Roumanie fût de nouveau en état de guerre avec l'Allemagne. Le 7/20 novembre 1918, les derniers soldats allemands quittent Buzău. La joie du Dr Bianu n'est assombrie que par son indignation contre leurs excès, cette fois-ci bacchiques, qui lui donnent l'occasion de rendre hommage à la dignité du peuple roumain, confiant même aux heures les plus difficiles dans le bien-fondé de sa propre cause - en contraste avec les soldats allemands, apparemment insensibles et peu intéressés au sort de leur pays:

«Nous voilà enfin débarrassés des Allemands. [...] Même au moment de partir ils ont refusé de se montrer civilisés. Ils n'ont pas dessoûlé depuis hier, braillant et faisant du foin [...]. À les voir, on dirait qu'ils se moquent bien de ce qui se passe chez eux; ils font preuve d'une inconscience révoltante. Quel Roumain aurait eu envie de faire la fête aux heures tristes et difficiles de la guerre?! Partout on ne voyait que des figures tristes et résignées, bien que nous soyons confiants dans le destin de notre nation et que nous attendions les temps meilleurs, les temps glorieux que nous vivons aujourd'hui » ${ }^{(72)}$.

Le 8/21 novembre 1918, l'armée roumaine faisait son entrée triomphale dans la ville.

Publiées après la fin de la guerre, les Notes du Dr Bianu s'apprêtent à mettre en valeur toute la signification patriotique d'une guerre considérée comme la guerre sainte pour l'union de tous les Roumains. Même si ces notices se rattachent explicitement à la pédagogie nationaliste, elles rendent compte également de toute une série de sentiments, d'émotions, d'impressions de leur auteur. Pour le Roumain originaire de la Transylvanie, l'acceptation de la guerre en tant que solution unique pour assurer la cohésion de la nation roumaine dans son intégralité va de soi. Pour le représentant de l'opinion publique, le rayonnement des grandes cultures européennes et le respect des valeurs fondamentales liées à la liberté des individus et des nations vont de pair; il n'y a pas de rapports possibles entre l'épanouissement culturel allemand, incontestable, et les ambitions de suprématie politique (voire militaire) de l'État allemand, considérées comme illégitimes. Pour le médecin, la guerre, bien que légitime, n’en reste pas moins meurtrière (il y perd des camarades ou des membres de sa propre famille). Le Dr Bianu enregistrait rigoureusement les événements internes et internationaux, les évolutions militaires, la situation médico-sanitaire des hôpitaux militaires, les conséquences de la guerre et de l'occupation pour la population civile, en interprétant toutes ces données à sa manière. On peut placer les Notes du Dr Bianu dans la perspective de la pédagogie nationale présentant même parfois des touches de mystique patriotique. Mais la sensibilité humanitaire du médecin, affirmée et assumée constamment par l'auteur, rend finalement très humaine cette construction savante. 


\title{
Résumé
}

Les Notes de la guerre de la Grande Roumanie publiées en 1926 par le Dr Vasile Bianu sont une monumentale construction idéologique qui trouve son prétexte dans le journal tenu par leur auteur au fil des événements. Le Dr Bianu envisage les rapports de la Roumanie avec les Puissances centrales sous l'angle de l'idéal national roumain, dissociant l'État allemand constitué par unification sur le principe des nationalités et l'État multinational austro-hongrois. Les évolutions dramatiques sur le front roumain détermineront une radicalisation du discours du Dr Bianu. Il dénonce le militarisme allemand, la contradiction entre la supériorité de la culture allemande et les horreurs de la guerre, les vols et les humiliations que les Allemands font subir aux Roumains. Outre la pédagogie nationaliste, Les Notes $d u$ Dr Bianu rendent compte également de toute une série de sentiments, d'émotions, d'impressions de leur auteur.

\section{Zusammenfassung}

Die Notizen aus dem Großrumäniens Krieg, die von Dr. Vasile Bianu im Jahre 1926 veröffentlicht wurden, sind eine monumentale ideologische Konstruktion, die auf dem Tagebuch basiert, das der Autor während der Geschehen aufbewahrt hat. Die Beziehungen Rumäniens zu den Mittelmächten werden aus der Perspektive des rumänischen nationalen Ideals betrachtet, wobei der Verfasser den deutschen Staat, der durch Vereinigung nach dem Nationalitätsprinzip gegründet wurde, vom multinationalen österreichisch-ungarischen Staat distanziert. Die dramatischen Entwicklungen an der rumänischen Front führten jedoch zur Radikalisierung von Dr. Bianus Diskurs. Er verurteilte den deutschen Militarismus, den Widerspruch zwischen der Überlegenheit der deutschen Kultur und den Schrecken des Krieges, den Diebstahl und die Demütigung, die die Rumänen von den Deutschen erlitten hatten. Aber jenseits der nationalistischen Pädagogik drückt Dr. Bianus Notizen auch eine ganze Reihe von Gefühlen, Emotionen, Eindrücken des Autors aus.

\begin{abstract}
The Notes from Great Romania's War, published by Dr. Vasile Bianu in 1926, are a monumental ideological construction, based on the diary kept by the author during the events. Romania's relations with the Central Powers are regarded from the perspective of the Romanian national ideal, the author dissociating the German state, established by unification on the nationality principle, from the multinational Austro-Hungarian state. However, the dramatic developments on the Romanian front led to the radicalization of Dr. Bianu's discourse. He denounced the German militarism, the contradiction between the superiority of the German culture and the horrors of the war, the theft and humiliation that Romanians have suffered from the Germans. But beyond the nationalist pedagogy, Dr. Bianu's Notes expresses also a whole range of feelings, emotions, impressions of the author.
\end{abstract}

